\title{
Multiobjective Evolutionary Algorithm for Physical Topology Design and Device Specifications of All-Optical Networks
}

\author{
Daniel A. R. Chaves, Carmelo J. A. Bastos-Filho and Joaquim F. Martins-Filho.
}

\begin{abstract}
In this paper we propose a methodology based on an evolutionary multiobjective optimization algorithm for all-optical network topology design. The aim is to define the topology layout and the specification of the optical devices that should be deployed in the network in order to minimize simultaneously the total installation cost of a communication network (capital cost) and the total network blocking probability (performance criteria). To accomplish that, we propose a capital cost model for the network. We considered the following physical layer impairments: losses in optical devices, amplified spontaneous emission in optical amplifier and homodyne crosstalk in optical cross connect (OXC), polarization mode dispersion (PMD) and residual dispersion (RD). The multiobjective optimization evolutionary algorithm used in the optimization process is the NSGA-II. Our proposed methodology can solve the network topology design problem taking into account the physical layer impairments and the capital costs simultaneously. We also present a case study to show the effectiveness of our methodology considering uniform and non-uniform network traffic.
\end{abstract}

Keywords-Physical network topology design, All-optical networks, physical impairments, multiobjective optimization, evolutionary algorithm.

\section{INTRODUCTION}

In all-optical networks, the signal remains in the optical domain between the edge nodes, i.e., the signal propagates along the core of the optical network without any optical-electronicoptical conversion [1]. Therefore, transmission physical impairments such as: amplified spontaneous emission noise (ASE), polarization mode dispersion (PMD), chromatic dispersion, crosstalk, self phase modulation (SPM) and four wave mixing (FWM) accumulate along the transmission of signal over the physical layer [2]. The signal degradation occurs because of the optical devices characteristics. For example, amplified spontaneous emission noise is generated as a result of the amplification process inside optical amplifiers, while crosstalk can occurs, among other devices, inside the optical crossconnects (OXC) [1]. The signal degradation that takes place in a specific optical device is related to its quality, and therefore, to its cost. Due to differences in the fabrication processes, the technology adopted or cost concerns, some devices impair more severely the optical signal than others. In this scenario, the network designer faces a challenge:

Daniel A. R. Chaves and Joaquim F. Martins-Filho are with the Department of Electronics and Systems, Federal University of Pernambuco, Recife, Brazil, 50740-530, E-mails: daniel.rchaves@ufpe.br, jfmf@ufpe.br

Carmelo J. A. Bastos-Filho, Department of Computing and Systems, University of Pernambuco, Recife, Pernambuco, Brazil, Email: cjabf@dsc.upe.br apply devices that are at the same time cost and performance efficient.

In addition, others concerns are reserved to the network designer such as: physical topology design [3], routing and wavelength assignment (RWA) strategy [4] , network survivability [1] among others. The problem of physical topology design is to determine which nodes of the network should be connected by means of physical optical link (i.e. optical fiber link). This choice must be made in a cost effective manner: if in one hand the network with many optical links has a high deployment cost, on the other hand, this network can handle a larger traffic demand. Other important topic in the design process is the choice of the RWA algorithm. In fact, since the RWA algorithm is responsible for the distribution of network traffic among the optical links, the selection of this algorithm can drastically change the optimal physical topology and vice-versa. Furthermore, the optical networks have been deployed by the telecommunications carriers as their network backbones. Consequently, they transport a large amount of data. Thus, a failure in a link or node of the network causes a significant loss of data, that must be avoided or mitigated. The designer must provide a survivable network, capable of handling these equipment failures by using either a re-routing strategy (restoration) or by means of backup routes (protection schemes) [1].

One can note that the design of an optical network is a multiobjective optimization problem over a multivariable design space [5]. It is multiobjective because the designer must satisfy, simultaneously, several performance constraints such as: maximum delay, capital costs, blocking probability, profits, traffic capacity, etc. To accomplish the design with these constraints, the designer must work in a multivariable design space in order to choose the network devices, routing algorithms, physical topology, node places, node degrees, etc. For these reasons the all-optical network topology design (NTD) considering physical layer aspects is an extremely hard problem.

Previous works in this field can be classified in two groups according to the techniques employed to solve the problem: those using ILP (Integer Linear Programming) or MILP (Mixed-Integer Linear Programming) formulations [6], and those using heuristics or metaheuristics [3] [7]. The first ones offer optimal solutions but they are time consuming for medium and big size networks, while the others are fast but only achieve suboptimal solutions. Besides, both groups can use either single optimization objective [7] (which is clearly a 
poor approach for the NTD problem) or multiple optimization objectives [8].

In this paper we propose a multiobjective optimization algorithm for network topology design (MOEA-NTD) to solve the physical network topology design problem for all-optical networks. To our knowledge, this is the first paper proposing to solve the network topology design problem taking into account the physical layer impairments and capital costs simultaneously. Section II describes the all-optical network topology design problem and the description of the network representation. Section III presents the physical layer model proposed by Pereira et al. [9] used in this paper. Section IV presents our proposal to evaluate the network capital cost. Section V describes the multiobjective evolutionary algorithm used to determine the network layout. Section VI presents the simulation setup and section VII shows the results. In section VIII we give our conclusions.

\section{Problem Description And Representation}

In this section we describe our formulation for the network topology design. We are concerned with the following problem: given the desired node locations, traffic matrix and RWA algorithm, the objective is to find the physical topology layout and the proper specification of the optical devices that should be deployed in the network in order to simultaneously minimize total network capital cost and total network blocking probability. Besides, the optimization process is constrained by the Quality of Service (QoS), traffic estimation and survivability requirements. To evaluate the network blocking probability, the physical layer model considers a minimum Optical Signal to Noise Ratio (OSNR) at the receiver in order to achieve a predetermined QoS for the lightpaths. It was assumed, as design variables, the following network parameters: topological layout, amplifier saturation power and noise figure in Erbium doped fiber amplifier (EDFA) in a per link basis, the isolation factor of all OXC in the network and the number of wavelength per link.

As input data, the nodes locations are defined as $P=$ $\left\{\left(x_{i}, y_{i}\right)\right\}(i \in 1,2, \ldots, N)$, where $x_{i}$ and $y_{i}$ are $x$ and $y$ cartesian coordinates of the $i$ th node, and $N$ is the total number of nodes. The traffic matrix and the RWA algorithm are also input variables as well. The node locations and traffic pattern are usual demands of telecommunication carriers. Therefore, we think it is fair to consider them as input variables in the optimization problem.

The first step in the network design process is to determine a proper representation for the physical topology. The most used form is the adjacency matrix. The adjacency matrix $A=$ $\left\{a_{i, j}\right\}$ is a boolean matrix which represents the connectivity between the network nodes. If $a_{i, j}=1$, the nodes labeled as $i$ and $j$ are connected by a physical link, otherwise $a_{i, j}=0$. In this work, we use a similar approach to represent network topology. It is given by a integer matrix $M=\left\{m_{i, j}\right\}\left(m_{i, j} \in\right.$ $0,1, \ldots L_{A}$ ). If $m_{i, j}=0$, the network nodes $i$ and $j$ are not connected, otherwise they are connected using one of the predetermined available types of optical amplifier in this link. The integer numbers $\left(1,2, . ., L_{A}\right)$ represent a label for a possible choice for the optical amplifiers and they will be defined in section IV. We consider that the network has a bidirectional link between the nodes, i.e. $m_{i, j}=m_{j, i}$. Since the $M$ matrix is symmetric $\left(m_{i, j}=m_{j, i}\right)$ one can represent this matrix in a vectorized form by merging side by side the lines in superior portion (relatively to the main diagonal) of the $M$ matrix. Therefore, this vector has $K=\frac{N^{2}-N}{2}$ entries. For the convenience of the optimization algorithm we append other two entries at the end of this vector. One to represent the choice of the OXC isolation factor $(\epsilon)$ parameter and the other to represent the number of wavelengths per link. Therefore, the network representation vector is defined as $V=\left\{v_{k}\right\}$ ( $k \in$ $1,2, \ldots, K+2)$, where the integer $v_{K+1}\left(v_{K+1} \in 1, \ldots, L_{S}\right)$ represents a label for a possible choice of the OXC device, and the integer $v_{K+2}\left(v_{K+2} \in W_{M I N}, \ldots, W_{M A X}\right)$ represents the number of wavelengths per link. $W_{M I N}$ and $W_{M A X}$ are the minimum and maximum allowed number of wavelengths per link. The k-th element of vector $V$ can be obtained from the $M$ matrix by using:

$$
v_{k}=m_{i, j}
$$

where $k=(j-i)+(i-1) N-\frac{i(i-1)}{2}, j>i$ and $k \neq$ $K+1, K+2$.

The relation between matrix $M$ and vector $V$ is such as:

$$
\begin{aligned}
& M=\left[\begin{array}{lllll}
m_{1,1} & m_{1,2} & m_{1,3} & m_{1,4} & m_{1,5} \\
m_{2,1} & m_{2,2} & m_{2,3} & m_{2,4} & m_{2,5} \\
m_{3,1} & m_{3,2} & m_{3,3} & m_{3,4} & m_{3,5} \\
m_{4,1} & m_{4,2} & m_{4,3} & m_{4,4} & m_{4,5} \\
m_{5,1} & m_{5,2} & m_{5,3} & m_{5,4} & m_{5,5}
\end{array}\right] \\
& V=\left[\begin{array}{llllllllllll}
m_{1,2} & m_{1,3} & m_{1,4} & m_{1,5} & m_{2,3} & m_{2,4} & m_{2,5} & m_{3,4} & m_{3,5} & m_{4,5} & \ell_{S} & W
\end{array}\right]
\end{aligned}
$$

Once the vector $V$ is defined, the goal of our algorithm is to find a set of optimized vectors $V$ (i.e. non-dominated solutions) using a multiobjective approach (explained in section $\mathrm{V}$ ) where the objectives are the network performance in terms of blocking probabilities (section III) and the network capital cost (section IV).

\section{Physical Layer Model}

The physical layer impairments are evaluated according the network architecture and model used in [9]. The optical signal to noise ratio (OSNR) of each lightpath is evaluated using that physical layer model and it considers the following impairments: ASE noise, amplifier gain saturation effect, saturation of ASE noise in EDFAs and homodyne crosstalk in optical switches. The four wave mixing (FWM) effect are not considered in the present work.

In addition to the model described in [9] we consider the residual chromatic dispersion impairment using the approach presented by Zulkifli et al. in [10].

The broadening of the optical pulses due to the residual 
dispersion is taken into account using:

$$
\begin{aligned}
\Delta t_{R D}=\Delta \lambda_{\text {transmitter }} & \sum_{j=i}^{i}\left\{\left[D_{T F}^{\lambda_{\text {ref }}}+\left(\Delta \lambda_{j} S_{T F}\right)\right] L_{T F_{j}}+\right. \\
& \left.+\left[D_{D C F}^{\lambda_{\text {ref }}}+\left(\Delta \lambda_{j} S_{D C F}\right)\right] L_{D C F_{j}}\right\},
\end{aligned}
$$

where

$$
L_{D C F_{j}}=\left|\frac{L_{T F_{j}} D_{T F}^{\lambda_{\text {ref }}}}{D_{D C F}^{\lambda_{\text {ref }}}}\right|
$$

$\Delta \lambda_{\text {transmitter }}$ represents the transmitter linewidth, $D_{T F}^{\lambda_{\text {ref }}}, \mathrm{S}_{T F}$ and $L_{T F_{j}}$ are the chromatic dispersion coefficient in the reference wavelength, the chromatic dispersion slope and the optical fiber length, respectively. $\Delta \lambda_{j}$ is the difference between the transmitter wavelength and the wavelength where the residual dispersion is zero.

Futhermore, we also consider the pulse broadening effect caused by PMD in a route using the following expression [2]:

$$
\Delta t_{P M D}=B \sqrt{\sum_{j=1}^{i} D_{P M D}^{2}(j) d(j)},
$$

where $B$ is the transmission bit rate, $D_{P M D}(j)$ is the PMD coefficient, and $d(j)$ is the length of the $\mathrm{j} t h$ link belonging to the lightpath.

The total pulse broadening $\Delta t$ is evaluate by:

$$
\Delta t=\Delta t_{R D}+\Delta t_{P M D}
$$

$\delta_{\%}$ represents the temporal broadening of the optical pulse, in percentage. It can be expressed by:

$$
\delta_{\%}=100 B \Delta t,
$$

where $B$ represents the transmission bit rate.

\section{Capital Cost Model}

The definition of a consistent WDM cost model comprising relevant network equipment is crucial for any technoeconomic study comparing different network alternatives. However, detailed cost values are hard to derive for many reasons such as: costs are usually commercially confidential, price variations and vendors discounts dependent on the operator [11]. In the design algorithm proposed in this paper, the capital costs of the devices are input parameters. It means that our approach do not depend on the prices of a specific manufacturer or another cost model.

In our capital cost model we consider four different sources of costs: a fixed cost for each wavelength used in the entire network, fiber deployment cost, optical amplifier cost and OXC cost. We defined a monetary unity (m.u.) that is used to evaluate the capital cost of each source of cost.

Each wavelength used in network has an associated capital cost due to the optical line terminal (OLT), i.e. an OpticalElectronic converter to interface the clients and the transparent network. The cost associated to the number of wavelength $\left(C O S T_{\text {Lambda }}\right)$ is given in m.u. and defined as:

$$
\operatorname{COST}_{\text {Lambda }}=\eta \cdot W
$$

where $W\left(W=v_{K+2}\right)$ is the number of wavelengths per link and $\eta$ is a constant value that can be inferred from the OLT equipment price.

The network has a set of links interconnecting the network nodes. There are two capital costs involved in this process, the cost of the fiber cable itself and the cable deployment. We included both costs in a unique function. Eq. 8 is used to evaluate the costs in m.u. per kilometer.

$$
\operatorname{COST}_{\text {Cable }}=2 \beta \sum_{i=1}^{N} \sum_{j=i+1}^{N} d_{i, j},
$$

where $d_{i, j}$ is the physical distance between the $i$ and $j$ nodes if they are connected and zero if they are not connected. $\beta$ is an input constant inferred from the equipment price. Since we use one fiber for transmission and other for reception, the sum evaluates the half of the total length of deployed fibers, thus we multiply it by 2 .

We consider the cost of the optical amplifiers according to two parameters: output saturation power and noise figure. Each saturation power and noise figure pair is labeled according Table I. The amplifier costs $\left(C O S T_{\text {Amplifier }}\right)$ is given by:

$$
\operatorname{COST}_{\text {Amplifier }}=2 \cdot \sum_{i=1}^{K} C_{a m p}\left(v_{i}\right),
$$

where $K=\frac{N^{2}-N}{2}, N$ is the number of nodes in the network and $v_{i}$ was defined by Eq. 1. $C_{a m p}\left(v_{i}\right)$ is depicted in Table I.

TABLE I

LABELS AND CAPITAL COST VALUES ADOPTED FOR DIFFERENT OPTICAL AMPLIFIER SPECIFICATION.

\begin{tabular}{cccl}
\hline Label $(\ell)$ & Output Saturation Power & Noise Figure & $\begin{array}{l}\text { Capital Cost } \\
\left(C_{a m p}(\ell)\right)\end{array}$ \\
\hline 1 & $13 \mathrm{dBm}$ & $8 \mathrm{~dB}$ & $1 \mathrm{~m} . u$. \\
2 & $13 \mathrm{dBm}$ & $5 \mathrm{~dB}$ & $2 \mathrm{~m} . u$. \\
3 & $16 \mathrm{dBm}$ & $8 \mathrm{~dB}$ & 3 m.u. \\
4 & $16 \mathrm{dBm}$ & $5 \mathrm{~dB}$ & 4 m.u. \\
\hline
\end{tabular}

We used a similar approach to define the OXC capital cost $\left(C O S T_{O X C}\right)$. However, the used OXC architecture [9], the node degree and the number of wavelengths used in the network are related to the OXC cost as well. As the node degree increases, the number of ports in the switches fabric used inside the OXC also increases. Furthermore, as the number of wavelengths increases, the number of switches inside the OXC also increases. Therefore, the total OXC capital cost is given by:

$$
\operatorname{COST}_{O X C}=\gamma \cdot C_{s w}\left(v_{K+1}\right) \cdot W \cdot \sum_{i=1}^{N} G(i),
$$

where $\gamma$ is related to the OXC equipment price, $G(i)$ is the node degree of the $i$ node and $C_{s w}(\ell)$ is related to the isolation factor and is defined in Table II. 
Now we can define the total network capital cost $\left(C O S T_{N e t}\right)$ as:

$$
\begin{array}{r}
C O S T_{\text {Net }}=C O S T_{\text {Lambda }}+C O S T_{\text {Amplifier }} \\
+C O S T_{\text {Cable }}+C O S T_{\text {OXC }} .
\end{array}
$$

TABLE II

LABELS AND CAPITAL COST VALUES ADOPTED FOR DIFFERENT OXC.

\begin{tabular}{ccc}
\hline Label $(\ell)$ & Isolation Factor $(\epsilon)$ & Capital Cost $\left(C_{s w}(\ell)\right)$ \\
\hline 1 & $-30 \mathrm{~dB}$ & $1 \mathrm{~m} . \mathrm{u}$. \\
2 & $-35 \mathrm{~dB}$ & 2 m.u. \\
3 & $-40 \mathrm{~dB}$ & $3 \mathrm{~m} . \mathrm{u}$. \\
4 & $-45 \mathrm{~dB}$ & 4 m.u. \\
\hline
\end{tabular}

\section{Multiobjective Optimization Algorithm For Network Physical Topology Design}

In this section we describe the multiobjective optimization algorithm used to find the physical topology layout and the optical devices specification in order to minimize simultaneously the network capital cost and the network blocking probability.

It has been shown that evolutionary algorithms can be used to efficiently solve multiobjective problems. To perform the multiobjective optimization, we used a multiobjective evolutionary algorithm (MOEA) called NSGA-II. The NSGA-II was proposed by Deb et al. [5] and is based on genetic algorithms (GA) [12]. The main difference between NSGA-II and an ordinary GA relies mainly on the selection operator. In the former, the selection is based on a dominance rule concerning the objectives involved in the optimization process.

In genetic algorithms, an individual represents a possible solution for the problem. In the NSGA-II, the vector $V$ is used as individuals, since it is able to represent all possible network configurations. Each entry in the vector that represents the individual is named gene, thus, $v_{k}$ is a gene.

In a regular GA, the quality of an individual is determined by its fitness. In MOEA the quality of an individual is related to the fitness in all objectives and a dominance criteria. Here, we evaluate the fitness of an individual using the capital cost model and the network blocking probability using the results from a simulation program that implements the physical layer model [9].

During each iteration genetic operators are used by the GA: crossover, mutation and selection. In this work the selection operator is performed using the Linear Ranking Selection (LRS) [13] in conjunction with Stochastic Universal Sampling (SUS) [12]. The crossover operator used was the uniform crossover [12]. The mutation is performed in a per gene basis and when a gene is selected to be mutated it is replaced by a new integer number raffled at random inside the gene limits.

A peculiar aspect of the NSGA-II relies in how a new population $Q_{t}$ is generated from the current population $P_{t}$ [5]. The approach used is based on the Make-New-Population paradigm. In this algorithm $P_{C}$ and $P_{M}$ are the crossover and mutation probabilities respectively.

\section{A. Unfeasible individuals and biconnectivity issue}

To allow the use of survivability strategies, such as protection or restoration, it is mandatory that all network nodes have at least two connections to different nodes (biconnectivity constraint) [14]. In addition, the graph that represents the network must be connected (i.e. always exists a possible route in the network from any source node to any other node of the network). If it does not occur, it means the network is divided in two or more pieces. It is desirable that the network satisfies both properties. Unfortunately the crossover and mutation operators used in this paper can generate individuals that violate these rules. To avoid this problem, we repair the individual before they join the new population. We do that by trying to add as few and as short links as possible to obey these constraint rules.

\section{Simulation SETUP}

Our simulation software for network blocking probability evaluations uses the following steps. Upon a call request it selects the route using Shortest Path algorithm (SP) [4] and the wavelength is defined using the first fit algorithm [4]. The route and wavelength define a lightpath. If no lightpath is found, i.e. there is no wavelength available, the call is blocked. If an available lightpath is found it is verified if this lightpath meets the QoS requirements. There are two verications to be performed: total pulse broadening and output OSNR. Firstly, the $\delta \%$ of the found lightpath is checked. If it is above the pre-determined level $(\delta)$, then the call request related to the lightpath is blocked. If the pulse broadening meets the QoS requirements (i.e. $\delta_{\%}<\delta$ ) the lightpath OSNR is verified. If the OSNR in the output of the lightpath is above the predetermined level $\left(O S N R_{Q o S}\right)$, then the call related to the lightpath is established. Otherwise the call is blocked. The blocked calls are lost. The blocking probability is obtained from the ratio of the number of blocked calls and the number of call requests.

Blocking probability of the network is a relative measure of how many communication requests are denied by the network due to either lack of physical resources or because the lightpath does not meet the QoS requirements. Therefore it is an important performance indicator.

For each network simulation a set of $10^{7}$ calls are generated by two different forms: an uniform traffic, characterized by choosing randomly (using an uniform distribution) the source-destination pair and the non-uniform traffic using the traffic matrix shown in Table III. This matrix was obtained from a random uniform generator. In both uniform and a non-uniform traffic the total network load is 200Erlangs and the calls time follow a Poisson process. We assume circuit switched bidirectional connections in two different fibers and no wavelength conversion capabilities. The position of the nodes utilized in this case study were: $P=\{(40.4,84.6), \quad(39.7,46.0), \quad(54.1,101.1), \quad(69.1,72.7)$ ,(87.6,75.5), (115.6,110.8), (111.8,72.3), (135.4,75.0), $(158.6,73.9), \quad(146.5,97.5), \quad(148.0,67.1), \quad(168.4,66.5)$, $(173.6,74.5),(167.4,79.0)\}$, where all the numbers are given in kilometers with the origin of the cartesian system 
arbitrarily placed. These points lead to a NSFNET shape network. The default simulation parameters are shown in Table IV.

TABLE III

TRAFFIC MATRIX USED FOR SIMULATE NON-UNIFORM TRAFFIC IN ERLANGS.

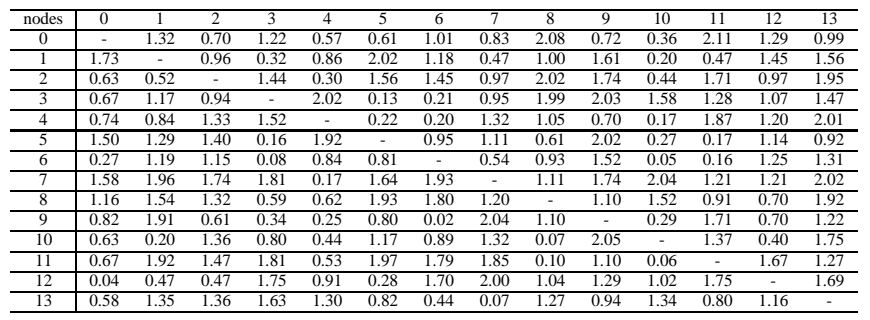

TABLE IV

DEFAULT SIMULATION PARAMETERS.

\begin{tabular}{|c|c|c|}
\hline Parameter & Value & Definition \\
\hline$O S N R_{i n}$ & $30 \mathrm{~dB}$ & Input optical signal-to-noise ratio. \\
\hline$O S N R_{Q o S}$ & $23 \mathrm{~dB}$ & $\begin{array}{l}\text { Optical signal-to-noise ratio for QoS cri- } \\
\text { terion. }\end{array}$ \\
\hline$B$ & $40 \mathrm{Gbps}$ & Transmission bit rate. \\
\hline$B_{o}$ & $100 \mathrm{GHz}$ & Optical filter bandwidth. \\
\hline$\Delta f$ & $100 \mathrm{GHz}$ & Channel spacing. \\
\hline $\bar{\lambda}_{i}$ & $1529.56 \mathrm{~nm}$ & The lower wavelength of the grid. \\
\hline$\lambda_{0}$ & $1557 \mathrm{~nm}$ & Zero dispersion wavelength. \\
\hline$\alpha$ & $0.2 \mathrm{~dB} / \mathrm{km}$ & Fiber loss coefficient. \\
\hline$L_{M u x}$ & $3 \mathrm{~dB}$ & Multiplexer loss. \\
\hline$L_{\text {Demux }}$ & $3 \mathrm{~dB}$ & Demultiplexer loss. \\
\hline$L_{\text {Switch }}$ & $3 \mathrm{~dB}$ & Optical switch loss. \\
\hline & $10 \%$ & Maximum pulse broadening. \\
\hline$D_{P M D}$ & $0.2 \mathrm{ps} / \sqrt{\mathrm{km}}$ & PMD coefficient. \\
\hline$W_{M I N}$ & 4 & $\begin{array}{l}\text { Minimum number of wavelengths per } \\
\text { link }\end{array}$ \\
\hline$W_{M A X}$ & 40 & $\begin{array}{l}\text { Maximum number of wavelengths per } \\
\text { link }\end{array}$ \\
\hline$F_{0}(\mathrm{NF})$ & $3.162(5 \mathrm{~dB})$ & Amplifier noise factor (Noise figure). \\
\hline $\mathrm{S}_{\mathrm{TF}}$ & $0.06 \mathrm{ps} / \mathrm{km} \cdot \mathrm{nm}^{2}$ & $\begin{array}{l}\text { Dispersion slope of the transmission } \\
\text { fiber (@1557 nm). }\end{array}$ \\
\hline$D_{\mathrm{DCF}}$ & $-1.87 \mathrm{ps} / \mathrm{km} . \mathrm{nm}$ & $\begin{array}{l}\text { Dispersion coefficient of the compensa- } \\
\text { tion fiber (@1541.35 nm). }\end{array}$ \\
\hline $\mathrm{S}_{\mathrm{DCF}}$ & $-126.18 \mathrm{ps} / \mathrm{km} \cdot \mathrm{nm}^{2}$ & $\begin{array}{l}\text { Dispersion slope of the compensation } \\
\text { fiber (@1541.35 nm). }\end{array}$ \\
\hline$\Delta \lambda_{\text {transmitter }}$ & $0.05 \mathrm{~nm}$ & Transmitter linewidth. \\
\hline$G$ & 1000 & Number of generations \\
\hline$P_{M}$ & 0.03 & Mutation probability \\
\hline$P_{C}$ & 1.0 & Crossover probability \\
\hline Pop & 50 & Population size \\
\hline$\eta_{\min }$ & 0.75 & LRS parameter \\
\hline$p$ & 50 & Number of selected individuals \\
\hline$\eta$ & 5 m.u. & Cost constant (wavelength cost) \\
\hline$\beta$ & $0.4 \mathrm{~m} . \mathrm{u} / \mathrm{km}$ & Cost constant (cable deployment cost) \\
\hline$\gamma$ & 0.2 m.u. & Cost constant (OXC cost) \\
\hline
\end{tabular}

\section{RESULTS}

The presence of multiple conflicting objectives in an optimization problem, in principle, implies in a set of optimal solutions (known as Pareto-optimal solutions), instead of a single optimal solution. In the absence of any further information, each point in these Pareto-optimal solutions cannot be said to be better than the other ones [5].

Using the values and assumptions described in section VI, we performed some simulations. Fig. 1 shows the simulation results for the network cost as a function of the obtained network blocking probability. In this case, we executed the NSGA-II algorithm for the two different network traffics scenarios previously defined in section VI: uniform and nonuniform traffic. Each symbol represents a optimized possible solution with its cost and blocking probability, i.e. each point corresponds to an specific network topology and device characteristics. One can note that the cost increases for lower blocking probabilities and vice versa. Using this figure, the network designer can choose the solution that meets his preferences, according to the project specification.

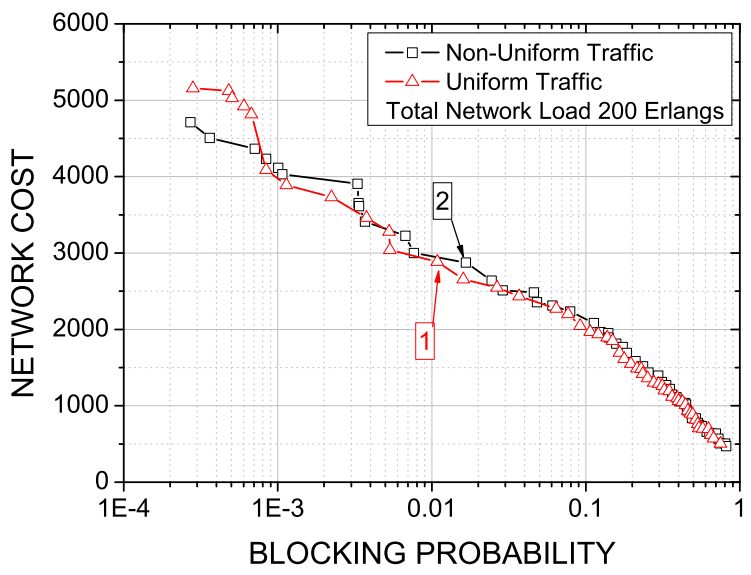

Fig. 1. Network cost as a function of the obtained network blocking probability for the two different traffics scenarios: uniform and non-uniform. The first Pareto front is shown for the both cases.

One can also note from the Fig. 1 that the best Pareto front found for non-uniform traffic (squares) and uniform (triangles) are very close except for very low blocking probability values (i.e. $B P<8 \times 10^{-4}$ ). It indicates that, for a given desired blocking probability, our algorithm is was able to design networks at almost the same cost independently of the traffic pattern.

Figs. 2 and 3 show examples of the network topologies and devices parameters found by the multiobjective algorithm respectively for uniform and non-uniform traffics. The numbers in parenthesis separated by commas represent the link lengths, output saturation power and noise figure of the amplifiers used in the link, respectively. The link length can be obtained from the position of the nodes given by the set $P$. As defined by Table I the optimization algorithm uses a label to represent the output saturation power and the noise figure of the amplifiers used in a given link. The labels found for Fig. 2 are shown in Table V and the labels found for Fig. 3 are shown in Table VI. The results shown in Tables V and VI corresponds to $M$ matrix found by the optimization process. Fig. 2 shows the network topology marked as 1 in Fig 1 and Fig. 3 shows the network topology marked as 2 in Fig 1 . The switch isolation and the number of available wavelength per fiber found for each topology is given in the figure caption. Comparing the figures one can note that in both cases the blocking probability and the network cost are very close. Despite of this, the network topologies are quite different. The algorithm changes the network physical topology design and device characteristics in order to accommodate the different traffic demands. 


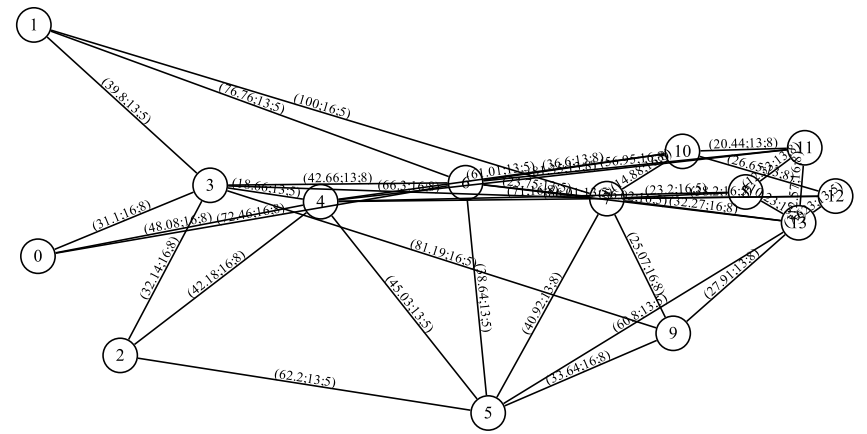

Fig. 2. The Network topology and devices parameters found for a blocking probability around $1 \%$ and uniform traffic $\left(B P=1.09 \%, C O S T_{N E T}=\right.$ 2887.05 m.u., $W=21, \epsilon=-45 d B$ ). Point number 1 in Fig. 1.

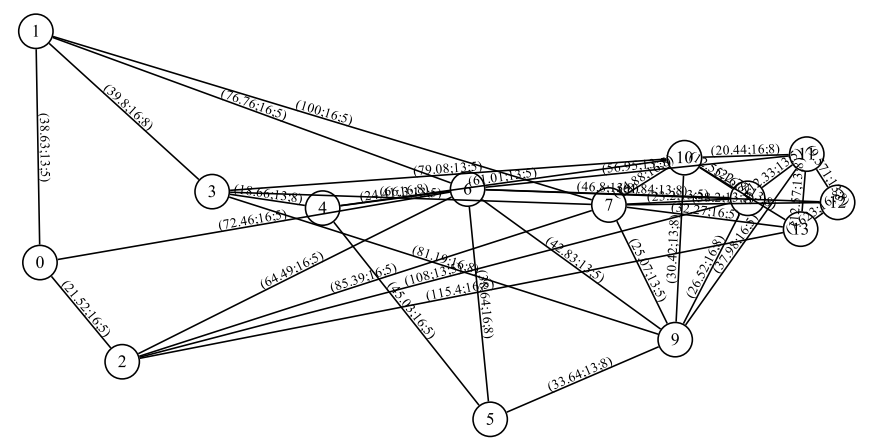

Fig. 3. The Network topology and devices parameters found for a blocking probability around $1 \%$ and non-uniform traffic $(B P=1.67 \%$, $C O S T_{N E T}=2874.17$ m.u., $W=20, \epsilon=-45 d B$ ). Point number 2 in Fig. 1.

TABLE V

THE $M$ MATRIX FOUND FOR THE TOPOLOGY SHOWN IN FIG. 2.

\begin{tabular}{c|cccccccccccccc}
\hline node & 0 & 1 & 2 & 3 & 4 & 5 & 6 & 7 & 8 & 9 & 10 & 11 & 12 & 13 \\
\hline 0 & 0 & 0 & 0 & 3 & 3 & 0 & 3 & 0 & 0 & 0 & 0 & 0 & 0 & 0 \\
\hline 1 & 0 & 0 & 0 & 2 & 0 & 0 & 2 & 4 & 0 & 0 & 0 & 0 & 0 & 0 \\
\hline 2 & 0 & 0 & 0 & 3 & 3 & 2 & 0 & 0 & 0 & 0 & 0 & 0 & 0 & 0 \\
\hline 3 & 3 & 2 & 3 & 0 & 2 & 0 & 1 & 3 & 0 & 4 & 0 & 0 & 0 & 0 \\
\hline 4 & 3 & 0 & 3 & 2 & 0 & 2 & 0 & 0 & 3 & 0 & 2 & 1 & 2 & 0 \\
\hline 5 & 0 & 0 & 2 & 0 & 2 & 0 & 2 & 1 & 0 & 3 & 0 & 0 & 0 & 2 \\
\hline 6 & 3 & 2 & 0 & 1 & 0 & 2 & 0 & 2 & 0 & 0 & 1 & 3 & 0 & 4 \\
\hline 7 & 0 & 4 & 0 & 3 & 0 & 1 & 2 & 0 & 4 & 3 & 3 & 0 & 4 & 3 \\
\hline 8 & 0 & 0 & 0 & 0 & 3 & 0 & 0 & 4 & 0 & 0 & 0 & 1 & 0 & 1 \\
\hline 9 & 0 & 0 & 0 & 4 & 0 & 3 & 0 & 3 & 0 & 0 & 0 & 0 & 0 & 1 \\
\hline 10 & 0 & 0 & 0 & 0 & 2 & 0 & 1 & 3 & 0 & 0 & 0 & 1 & 1 & 0 \\
\hline 11 & 0 & 0 & 0 & 0 & 1 & 0 & 3 & 0 & 1 & 0 & 1 & 0 & 0 & 3 \\
\hline 12 & 0 & 0 & 0 & 0 & 2 & 0 & 0 & 4 & 0 & 0 & 1 & 0 & 0 & 2 \\
\hline 13 & 0 & 0 & 0 & 0 & 0 & 2 & 4 & 3 & 1 & 1 & 0 & 3 & 2 & 0 \\
\hline
\end{tabular}

TABLE VI

THE $M$ MATRIX FOUND FOR THE TOPOLOGY SHOWN IN FIG. 3.

\begin{tabular}{c|cccccccccccccc}
\hline node & 0 & 1 & 2 & 3 & 4 & 5 & 6 & 7 & 8 & 9 & 10 & 11 & 12 & 13 \\
\hline 0 & 0 & 2 & 4 & 0 & 0 & 0 & 4 & 0 & 0 & 0 & 0 & 0 & 0 & 0 \\
\hline 1 & 2 & 0 & 0 & 3 & 0 & 0 & 4 & 4 & 0 & 0 & 0 & 0 & 0 & 0 \\
\hline 2 & 4 & 0 & 0 & 0 & 0 & 0 & 4 & 4 & 2 & 0 & 0 & 0 & 0 & 4 \\
\hline 3 & 0 & 3 & 0 & 0 & 1 & 0 & 0 & 2 & 0 & 3 & 2 & 0 & 0 & 0 \\
\hline 4 & 0 & 0 & 0 & 1 & 0 & 4 & 3 & 0 & 0 & 0 & 2 & 0 & 0 & 0 \\
\hline 5 & 0 & 0 & 0 & 0 & 4 & 0 & 3 & 0 & 0 & 1 & 0 & 0 & 0 & 0 \\
\hline 6 & 4 & 4 & 4 & 0 & 3 & 3 & 0 & 0 & 1 & 2 & 0 & 2 & 1 & 0 \\
\hline 7 & 0 & 4 & 4 & 2 & 0 & 0 & 0 & 0 & 2 & 2 & 3 & 0 & 2 & 4 \\
\hline 8 & 0 & 0 & 2 & 0 & 0 & 0 & 1 & 2 & 0 & 3 & 3 & 2 & 0 & 0 \\
\hline 9 & 0 & 0 & 0 & 3 & 0 & 1 & 2 & 2 & 3 & 0 & 1 & 4 & 0 & 0 \\
\hline 10 & 0 & 0 & 0 & 2 & 2 & 0 & 0 & 3 & 3 & 1 & 0 & 3 & 0 & 1 \\
\hline 11 & 0 & 0 & 0 & 0 & 0 & 0 & 2 & 0 & 2 & 4 & 3 & 0 & 1 & 1 \\
\hline 12 & 0 & 0 & 0 & 0 & 0 & 0 & 1 & 2 & 0 & 0 & 0 & 1 & 0 & 3 \\
\hline 13 & 0 & 0 & 4 & 0 & 0 & 0 & 0 & 4 & 0 & 0 & 1 & 1 & 3 & 0 \\
\hline
\end{tabular}

\section{CONCLUSIONS}

In this paper we proposed a multiobjective algorithm to solve the physical network topology design problem for all- optical networks. We considered capital cost and network performance in terms of blocking probability as the optimization objectives. The network performance is infered considering physical layer impairments. A case study was performed and the simulation results show that the methodology was successful in obtaining the network topology and optical devices parameters for different scenarios. Furthermore, it allows the network designer to choose, among an optimized set, which specific network will be implemented. It is a very powerful tool to analize an important network design trade-off (cost versus network performance).

It is worth noting that the proposed methodology and algorithm for multiobjective optimization presented here can be used with other capital cost models and other network performance parameters. It is not limited to the ones presented here.

\section{ACKNOWLEDGMENT}

The authors acknowledge the financial support from FACEPE, CNPq and CAPES.

\section{REFERENCES}

[1] R. Ramaswami and K. N. Sivarajan, Optical Networks: A Practical Perspective, 2nd ed. Morgan Kaufmann, 2002.

[2] J. Strand, A. Chiu, and R. Tkach, "Issues for routing in the optical layer,' Communications Magazine, IEEE, vol. 39, no. 2, pp. 81-87, Feb 2001.

[3] M. Gerla and L. Kleinrock, "On the topological design of distributed computer networks," IEEE Transactions on Communications, vol. 25 , no. 1, pp. 48-60, Jan 1977

[4] H. Zang, J. P. Jue, and B. Mukherjee, "A review of routing and Wavelength assignment approaches for wavelength-routed optical WDM networks," SPIE/Kluwer Publishers Optical Networks Magazine, vol. 1, no. 1, pp. 47-60, January 2000.

[5] K. Deb, A. Pratap, S. Agarwal, and T. Meyarivan, "A fast and elitist multiobjective genetic algorithm: NSGA-II," IEEE Transactions on Evolutionary Computation, vol. 6, no. 2, pp. 182-197, Apr 2002.

[6] R. Dutta and G. N. Rouskas, "A survey of virtual topology design algorithms for wavelength routed optical networks," Optical Networks, vol. 1, pp. 73-89, 2000.

[7] K.-T. Ko, K.-S. Tang, C.-Y. Chan, K.-F. Man, and S. Kwong, "Using genetic algorithms to design mesh networks," Computer, vol. 30, no. 8 , pp. 56-61, Aug 1997.

[8] R. t. Duran, "Multipurpose genetic algorithm based in pareto optimality to design logical topologies in reconfigurable wdm networks," ICTON 2006, vol. 4, pp. 27-30, June 2006.

[9] H. A. Pereira, D. A. R. Chaves, C. A. Bastos-Filho, and J. F. Martins-Filho, "OSNR model to consider physical layer impairments in transparent optical networks," Photonic Network Communications. [Online]. Available: http://dx.doi.org/10.1007/s11107-008-0178-2

[10] N. Zulkifli, R. C. Almeida-Jr, and K. M. Guild, "Efficient resource allocation of heterogeneous services in transparent optical networks," Journal of Optical Networking, vol. 6, no. 12, pp. 1349-1359, December 2007.

[11] M. Gunkel et al. , "A cost model for the wdm layer," International Conference on Photonics in Switching, 2006. PS '06., pp. 1-6, Oct. 2006.

[12] K. Man, K. Tang, and S. Kwong, "Genetic algorithms: concepts and applications [in engineering design]," IEEE Transactions on Industrial Electronics, vol. 43, no. 5, pp. 519-534, Oct 1996.

[13] F. Herrera and M. Lozano, "Gradual distributed real-coded genetic algorithms," IEEE Transactions on Evolutionary Computation, vol. 4, no. 1, pp. 43-63, Apr 2000.

[14] Y. Xin, G. N. Rouskas, and H. G. Perros, "On the physical and logical topology design of large-scale optical networks," Journal of Lightwave Technology, vol. 21, pp. 904-915, 2003. 